parallax $(\pi)$ of a star can be immediately deduced if its velocity (V) in miles per second in the line of sight has been determined by observation. The advantages to be expected from spectrographic observations of double stars for which $\pi \mathrm{V}$ is greater than $O^{\circ} \mathrm{I}$ are shown to be :-

(I) An independent check on the parallax where this has been determined trigonometrically.

(2) A determination of the parallax where, owing to its smallness, the trigonometrical method fails.

(3) A determination of the sign of the inclination which will remove the ambiguity attaching to the situation of the orbit.

It is to be hoped that astronomers who have the requisite instruments for this kind of observation may be induced to take up what appears to be a promising field of work.

TURIN OBSERVATORY.---We have received various publications from the Observatory of the Royal University of Turin. Amongst them we find convenient ephemerides of the sun and moon for 1889 and for 1890 calculated for the horizon of Torino by Señors Porro and Aschieri respectively, and a note by the former observer on the total eclipse of the moon on January 28 I888. The difference of longitude between the meridian circl at Turin Observatory and Milan Observatory has also been redetermined. The value found in 1823 was $5 \mathrm{~m}$. $58 \cdot 85 \mathrm{~s}$., and the value now found is $5 \mathrm{~m} .58 \cdot 736 \pm 0.006$; thus the difference between the two observations is only O.IIs, although the former was not made by telegraphy.

\section{A CONTRIBUTION TO THE ETIOLOGY OF} DIPHTHERIA. ${ }^{1}$

THE microbe, which was first described by Klebs (at the Wiesbaden Congress in 1883 ), then isolated and grown in artificial cultures by Löffler (Mitth. aus dem K'. Gesundheitsante, vol. ii.) from human diphtheritic membrane, was shown by this observer to act virulently on various animals. The Klebs-Löffler bacillus-by which name the diphtheria microbe is known-is the one with which also Roux and Yersin (Annales de l'Institut Pasteur, ii., 1888, No. 12) obtained positive results on guineapigs.

In the Reports of the Medical Officer of the Local Government Board for 1888-89 and 1889-90, I have shown that there occur in diphtheritic membranes two species of bacilli, very similar in morphological respects, and also in cultures on serum and on agar, but differing from one another in this, that one species, Klebs-Löffler bacillus No. I, is not constant in diphtheritic membranes, does not grow on solid gelatine at $19^{\circ}-20^{\circ} \mathrm{C}$., and does not act pathogenically on animals; the other species, KlebsLöfller bacillus No. 2, is constant in diphtheritic membranes, in fact is present even in the deeper layers of the membranes in great masses and almost in pure culture, acts very virulently on animals, and grows well on gelatine at $19^{\circ}-20^{\circ} \mathrm{C}$. Löfler, and after him other observers (Flügge, "Dle Mikroorganismen," 1886), considered it as a character of the diphtheria bacillus that it does not grow on gelatine below $22^{\circ}$ C., but this character, though true of the Klebs-Löffler species No. 1 , does not appertain to the diphtheria bacillus species No. 2. In fact, there is no difficulty in obtaining pure cultures of this bacillus on gelatine if a particle of diphtheritic membrane be taken and well shaken in two or three successive lots of sterile salt solution, and from the last lot plate cultivations on gelatine are made. In this way I have obtained the diphtheria bacillus in great numbers of colonies and in pure culture. Zarniko (Centralbl.f. Bakteriol. u. Parasit., vol. vi., 1889, p. 154) and Escherich (ibid., vol. vii., 189o, p. 8) both state that the diphtheria bacillus does grow on gelatine below $20^{\circ} \mathrm{C}$.

This bacillus diphtherix acts very virulently on guinea-pigs on subcutaneous inoculation; at the seat of the injection a tumour is produced, which in its pathology and in microscopic sections completely resembles the diphtheritic tissue of the human. In human diphtheria the diphtheria bacillus is presen only in the diphtheritic membrane, but neither in the blood nor in the diseased viscera; the same holds good for the experimental guinea-pigs. In subcutaneous inoculation with artificia culture, though it causes in these animals acute disease and

${ }^{1}$ Paper read before the Royal Society by Dr. E. Klein, F.R.S., on May 22. This research was undertaken for the Medical Department of the Local Government Board, and was communicated to the Royal Society with the permission of the Medical ufficer.

NO. IO74, VOL. 42$]$ death-the lungs, intestine, and kidney are greatly congested--
the diphtheria bacillus remains limited to the seat of inoculation. It was for these reasons that Löffler concluded that in diphtheria the diphtheritic membrane alone is the seat of the multiplication of the diphtheria bacillus, and that here a chemical poison is produced, which absorbed into the system causes the general diseased condition and eventually death. Roux and Yersin have then separated from artificial broth cultures the bacilli and the chemical products, and, by the injection of these latter alone into guinea-pigs, have produced a general effect. I have in this year's Report to the Medical Officer of the Local Government Board $(1889-90)$ shown that in these experiments of injection of cultures into guinea-pigs, an active multiplication of the diph. theria bacilli at the seat of inoculation can be demonstrated by culture experiments ; from the local diphtheritic tumour and the nearest lymph glands the diphtheria bacilli can be obtained in pure culture on gelatine.

On various occasions during the last three years information has reached me by Health Officers (Dr. Downes, Mr. Shirley Murphy, Dr. Thursfield) as to a curious relation existing between a mysterious cat disease and human diphtheria in this manner, that a cat or cats were taken ill with a pulmonary disease, and while ill were nursed by children, and then these latter sickened with well-marked diphtheria. Or children were taken ill with diphtheria, and either at the same time or afterwards the cat or cats sickened. The disease in the cat was described as an acute lung trouble; the animals were quiet, did not feed, and seemed not to be able to swallow; in some cases they recovered, in others they became emaciated, while the lung trouble increased, and ultimately they died. In one instance--in the north of London, in the spring, I889-this cat malady, occurring in a house where diphtheria soon afterwards appeared amongst the children, was of a widespread nature; a veterinary surgeonMr. Daniel-informed me that at that time he had several patients amongst cats affected with the disease, consisting in an acute catarrhal affection, chiefly of the respiratory passages. $\mathrm{He}$ furnished me with two such animals : one that after an illness of several weeks had died, another that was sent to me in a highly emaciated state, affected with severe broncho-pneumonia; this animal was paralyzed on the hind limbs. In both instances the post-mortem examination showed severe lung disease, bronchopneumonia, and large white kidneys due to fatty degeneration of the entire cortex. A similar condition is met with in the human subject in diphtheria. Further, I received from Dr. Thursfield, of Shrewsbury, the body of a cat that had died after a few days illness from pneumonia in a house in which children were ill with diphtheria; another cat in the same house that became next ill with the same lung trouble also succumbed. The post-mortem examination of the animal that I received showed severe bronchopneumonia and large white kidneys, the entire cortex being in a state of fatty degeneration.

Subcutaneous inoculations of cats were carried out with particles of fresh human diphtheritic membranes and with cultures of the diphtheria bacillus (Report of the Medical Officer of the Local Government Board, I 889-90); hereby a local diphtheritic tumour was produced at the seat of inoculation, and a general visceral disease; in the cases in which death followed after a few days the lungs were found much congested; when death followed after one or more weeks, the lungs showed broncho-pneumonia and the kidneys were enlarged and white, the cortex being in a state of fatty degeneration; if the disease in the animals lasted beyond five to seven days, both kidneys were found uniformly white in the cortex; if of shorter duration, the fatty degeneration was sometimes only in patches. Although in these experiments the bacillus diphtheria was recoverable by cultivation from the diphtheritic tumour at the seat of inoculation, there were no bacilli found in the lungs, heart's blood, or kidney, and the conclusion is justified that, just as in the human diphtheria and in the diphtheria produced by subcutaneous inoculation in the guinea-pig, so also in these experimental cats the visceral disease must be a result of the action of a chemical poison produced by the diphtheria bacillus at the seat of inoculation.

From this it is seen that the similarity between the artificial disease and the natural disease in the cat is very great, and the question that presents itself is, In what manner does the animal receive or give the diphtheritic contagium in the natural disease? The natural disease in the cat is in its symptoms and pathology a lung disease, and it is reasonable to suppose from analogy that the lung is the organ in which the diphtheritic process in the cat has its seat. The microscopic examination of the diseased lung 
of cats that died from the natural disease bears this out, the membrane lining the bronchi in the diseased portions of the lobules presenting appearances which in microscopic character coincide with the appearances in the mucous membrane of the human fauces, pharynx, or larynx in diphtheria. But the correctness of the above supposition, that diphtheria has its seat in the lung of the cat naturally diseased, was proved by direct experiment. Broth culture of the bacillus diphtheriæ was introduced into the cavity of the normal trachea without injuring the mucous membrane. The animals became ill with acute pneumonia, and on post.mortem, two to seven days after, there was found extensive pneumonia, and fatty degeneration of the kidney. The bronchi, infundibula, and air-cells of the inflamed lobules were found occluded by, and filled with, exudation, which under the microscope bears a striking resemblance to human diphtheritic membranes, and in the muco-purulent exudation in the large bronchi and trachea the diphtheria bacilli were present in large numbers.

During the last ten or twelve years certain epidemics of diphtheria have occurred which were traced to milk, but the manner in which that milk had become contaminated with the diphtheritic virus could not be demonstrated, although the evidence as to the milk not having been directly polluted from a human diphtheria case was very strong. The epidemic of diphtheria that prevailed in the north of London in 1878 , investigated by Mr. Power for the Local Government Board, then the epidemic that occurred in October I886 at York Town and Camberley, the epidemic in Enfield at the beginning of 1888 , and in Barking towards the autumn of 1888 , were epidemics of this character. Mr. Power, in his Report to the Local Government Board on the York Town and Camberley outbreak, states (p. 13) that a veterinary surgeon had certified that the cows from whom the infected milk was derived were all in good health, but that two of the cows showed "chaps" on their teats, and he adds that even two or three weeks after the epidemic had come to an end -the use of milk having been in the meanwhile discontinuedhe saw at the farm one cow which had suffered chapped teats. At Enfield a veterinary inspector had also certified that the cows were in good health; but at Barking the veterinary inspector found sores and crusts on the udder and teats of the cows.

I have made experiments at the Brown Institution on milch cows with the diphtheria bacillus, which appear to me to throw a good deal of light on the above outbreaks of diphtheria.

Two milch cows ${ }^{1}$ were inoculated with a broth culture of the diphtheria bacillus derived from human diphtheria. In each case a Pravaz syringeful was injected into the subcutaneous and muscular tissue of the left shoulder. On the second and third days there was already noticed a soft but tender swelling in the muscle and the subcutaneous tissue of the left shoulder; this swelling increased from day to day, and reached its maximum about the end of the week; then it gradually became smaller but firm. The temperature of both animals was raised on the second and third day, on which days they left off feeding, but after this becarne apparently normal. Both animals exhibited a slight cough, beginning with the eighth to tenth day, and this gradually increased. One animal left off feeding and ruminating on the twelfth day, "fell in " considerably, and died in the night from the fourteenth to fifteenth day; the other animal on the twenty-third to twenty-fourth day left off taking food, "fell in " very much, and was very ill : it was killed on the twenty-fifth day.

In both animals, beginning with the fifth day, there appeared on the skin of the udder, less on the teats, red raised papules, which in a day changed into vesicles, surrounded by a rim of injected skin; the contents of the vesicles was a clear lymph, the skin underneath was much indurated and felt like a nodule next day the contents of the vesicle had become purulent, i.e. the vesicle had changed into a pustule; in another day the pustule dried into a brownish-black crust, with a sore underneath this crust became thicker and larger for a couple of days, then became loose, and soon fell off, a dry healing sore remaining underneath. The whole period of the eruption of papules, leading to vesicles, then to pustules, and then to black crusts, which, when falling off, left a dry healing sore behind, occupied from five to seven days. The eruption did not appear in one crop : new papules and vesicles came up on the udder of one cow almost daily between the fifth and eleventh day after inoculation, in the other cow

I The cows had been kept under observation previous to the experiment for ten days, and were in all respects perfectly normal.

NO. IO74, VOL. 42$]$ between the sixth and tenth day; the total number of vesicles in the fomer cow amounted to about twenty-four on the udder, four on the teats; in the latter they were all on the udder, and amounted to eight in all. The size of the vesicles and pustules differed : some were not more than $\frac{1}{5}$ th of an inch, others larger, up to $\frac{1}{2} \cdot \frac{3}{4}$ of an inch in diameter; they had all a rounded outline, some showed a dark centre. From one of the above cows on the fifth day milk was received from a healthy teat, having previously thoroughly disinfected the outside of the teat and the milker's hand; from this milk cultivations were made, and it was found that thirty-two colonies of the diphtheria bacillus without any contamination were obtained from one cubic centimetre of the milk.

Unlike in the human, in the guinea-pig and in the cat the diphtheria bacillus passed from the seat of inoculation into the system of the cow; this was proved by the demionstration of the diphtheria bacillus in the milk. But also in the eruption on the udder, the presence of the diphtheria bacillus was demonstrated by microscopic specimens and particularly by experiment. With matter taken from the eruption-vesicles and pustules-of the udder, two calves were inoculated into the skin of the groin; here the same eruption made its appearance: red papules, rapidly becoming vesicular, then pustular, and then became covered with brown-black crusts, which two or three days after became loose and left a dry healing sore behind. More than that, the calves that showed this eruption after inoculation became affected with severe broncho-pneumonia and with fatty degeneration of the cortex of the kidney. In the two cows above mentioned, on post-mortem examination, both lungs were found highly congested, œdematous, some lobules almost solid with broncho-pneumonia in the upper lobes and the upper portion of the middle or lower lobe respectively; the pleural lymphatics were filled with serum and blood. Hæmorrhages in the pericardium and lymph glands, and necrotic patches were present in the liver. At the seat of inoculation there was in both cases a firm tumour consisting in necrotic diphtheritic change of the muscular and subcutaneous tissue. In this diphtheritic tumour continuous masses of the diphtheria bacillus were present ; their gradual growth into and destruction of the muscular fibres could be traced very clearly.

It appears then from these observations that a definite disease can be produced in the cow by the diphtheria bacillus, consisting of a diphtheritic tumour at the seat of inoculation with copious multiplication of the diphtheria bacillus, a severe pneumonia, and necrotic change in the liver; the contagious nature of the vesicular eruption on the udder and excretion of the diphtheria bacillus in the milk prove that in the cow the bacillus is absorbed as such into the system.

From the diphtheritic tumour, by cultivation, pure cultures of the diphtheria bacillus were obtained; a small part removed from the tumour with the point of a platinum wire, and rubbed over the surface of nutrient gelatine or nutrient agar, yielded innumerable colonies of the diphtheria bacillus without any contamination. In cultural characters in plate, streak, and stab cultures and in cover-glass specimens of such cultures, this cow diphtheria bacillus coincided completely with the human diphtheria bacillus, but in sections through the diphtheritic tumour of the cow a remarkable difference was noticed between it and the bacillus from the cultures; inasmuch as in the tissue of the tumour the masses of the microbe, both in the necrotic parts, as also where growing into and destroying the muscular fibres, were made up of filaments, granular threads, some of which possessed terminal oval or flask-shaped swellings. But that it was really the diphtheria bacillus was proved by culture experiments and by cover-glass specimens. In the latter, the transitional forms between typical diphtheria bacillus and long filaments with terminal knob-like swellings, with spherical or oblong granules interspersed here and there in the threads, could be easily ascertained. In the large number of cultivations that were made of the fresh tumour in both cows, the colonies obtained were all of one and the same kind, viz, those of the diphtheria bacillus ; no contamination was present in any of the cultivations.

Appendix, May 20.--At the beginning of the month of April two cats died at the Brown Institution, after having been ill for several days, with symptoms like those of natural cat diphtheria. Between the beginning of April and the beginning of May, 14 cats became similarly affected, some more severely than others, and some died with the characteristic morbid change 'This epidemic, as it may be called, commenced with the illness of the 
first two cats about the end of March ; and the question arises as to how the disease originated in these two animals. No cats had been ill in their shed, and the two affected ones were healthy when received at the institution some weeks before. But during the latter half of March there were in the stables of the institution two milch cows ill with diphtheria induced by inoculation with the human diphtheria bacillus-in fact, the two cows already referred to. The diphtheria bacillus was found in the milk drawn from one of these animals on the fifth day after inoculation, and orders were given to the attendant that the milk of both cows was to be thrown away. This order was not obeyed, for part of the milk was given to the two cats above mentioned, and they sickened as described within a day or two afterwards. It ought to be mentioned that the man in attendance on the cows bad also charge of the cats, but, in view of the fact that he was himself free from the disease, the possibility of his having conveyed it from the cows to the cats may be disregarded.

\section{SOME NOTES FROM SOUTH AMERICA.}

$\mathrm{I}$ the course of a visit to the plains of South America, not far from Rosario de Santa Fe, in the Argentine Republic, lasting from September $x 888$ until March 1880 , I was able to make some miscellaneous notes of more or less interest. From these I select the following :-

(I) The Rhea, or South American Ostrich.-The cock bird makes the nest, hatches the eggs, and takes care of the young birds. We had some (so-called) "tame" ostriches about the cstancia. One day I came across the old cock in a nest that it had made in the dry weeds and grass. Its wings and feathers were loosely arranged, and looked not unlike a heap of dried grass; at any rate the bird did not attract my attention until I was close on him. The long neck was stretched out close along the ground ; the crest-feathers were flattened; and an appalling hiss greeted my approach. It was a pardonable mistake if for a moment I thought I had come across a huge snake, and sprang back hastily under this impression. This might be cited as an instance of (unconscious) "protective mimicry."

When a troop of these birds is alarmed while yet at a distance from the enemy, they run with their wings either close to the side in the normal position, or raised above the back into a narrow wedge that offers but little resistance to the air. But when a bird is somewhat pressed, it usually droops the wings loosely, almost trailing them. And when in danger of being caught by dogs, or struck by the bolas of a horseman, it begins to dodge and twist in a very curious manner, the wings assuming various positions. It would seem as though the wings, thus used, may help the bird to make its sudden halts and turns; and also, when dogs are used in the chase, to baftle the attacks of these enemies. It was very curious to see the "tame" ostriches indulying in these freaks even when unpursued by the dogs of the estancia. The birds would rush straight along, turn and twist, contort their necks into very comical shapes, jump, and not unfrequently tumble over in their efforts to perform some unusually complicated evolution.

I may add that in the course of some years of " ostrichrunning," my brother once observed a troop of these birds swim a river that crossed their path. He himself followed, and found that the river was really out of their depth; they were not wading.

(2) Snakes: the "Vivora de la Cruz."-On October 6, 1888, we came across one of these common poisonous snakes, probably not long roused from its winter torpor. The dogs stood round it barking; and it remained, threatening a strike. With its tail (and against the grass?) it made a very distinct though not a loud burring, vibrating sound. This, my brother told me, was usual. Yet there is no kind of "rattle" on the tail.

At the end of March 1889, after I had returned to England, my brother killed a large vivora de la cruz; and, observing that it appeared to be very thick in the body, he cut it open in order to examine it. Inside was a string of transparent bags, six or seven in number, connected with one another. In each of these could be seen a fully-formed young snake, about 6 inches long, as far as he could say without exact measurement, coiled up. Two of the bags he cut open ; and the young snakes, released, both threatened to strike anything that approached them, and made, though of course on a very small scale, the vibrating, burring noise with the tail.
(3) The Intelligence (?) of Ants.-One kind of small ant, if not more, makes large nests underground, in the shorter grass. A network of paths, clear of grass, about 2 inches wide near the nest, converge towards this latter; being the roads by which the ants bring home forage. These paths are of all lengths from 10 yards up to 100 yards; and, as one traces them further from the nest, they break up into smaller branches and are finally lost. As a general rule, one may say that streams of ants carrying leaves, buds, flowers, seeds, and other valuable odds and ends are always moving towards the nest, while emptymouthed ants are meeting and passing them on their outward journey to the foraging grounds. Having, however, noticed a few burdened ants proceeding with great difficulty against the general stream of their burdened fellow-citizens, I tried the experiment of turning some of these carriers round when they had nearly reached home. The general conclusion I came to was that these ants did not then understand in what direction the nest lay, nor did they (as far as I could see) draw any con. clusions from the fact that they now met the stream of carriers with which they had previously been travelling.

Thus, one ant, carrying a (relatively) huge burden, I reversed in direction when already near the nest. I then followed it for about 8 yards (or about 20 minutes of time as far as I can say) in its mistaken reversed course away from the nest. Though it met and collided with quantities of burdened ants, and was passed in the same direction as its own by unburdened ants only, it did not seem to take the hint. Its final return home was the result of accident, as far as I could tell; it having got up "the right way round" after a severe fall.

Still it must be noticed that among the undisturbed ants very few went the wrong way.

I dug a hole in one of the paths, on several occasions. The hole was small ; and it was easy, though not so convenient, to go round by the side over the very short grass. Nevertheless it required the falling of very many ants into the hole, and the leaving of quite a pile of leaves there, before the stream learned to pass about about one inch to one or other side, and so to avoid the pitfall. Some ants even turned back; and I left them carrying their burdens back to the foraging grounds again.

(4) Grasses.-I noticed two grasses concerning whose seeds a remark or two may be of interest.

(a) One is called "Flechilla." Its seed bears a very sharp point; and a number of hairs, turned back from the point, prevent the return of the seed from any body into which it has penetrated. Attached to the seed is always a piece of stem curiously twisted like rope. The whole answers somewhat to an arrow-head with barbed point and with shaft attached. My brother, whose observations extend over more than twenty years, tells me that this seed penetrates into the bodies of sheep, and is found in their internal organs. The spring lambs, which are left unshorn until summer is over, are especially troubled with the flechilla. When one of these animals dies and is skinned, it is very commonly found that quantities of these seeds have penetrated the skin, the heads being found in the flesh underneath; and my brother has found them in the liver. It is believed that this is the cause of some deaths among the animals.

(B) Another remarkable grass is the "Paja voladora." This grows in tufts, not unlike those of a small "Pampas grass." "From slender stems there stand out still slighter branches, at the end of which are the seeds; the whole, stem and seeds, having somewhat the appearance of a miniature firtree as regards shape, and having various lengths up to 2 feet or so. When ripe, these stems with the seeds blow bodily away in the first strong wind. I have seen them flying through the air, looking from afar rather like a dust-cloud against the sky; and half rolling, half drifting, over the living grass of the plain, before the sudden onset of a tormenta (storm with wind). This drifting movement over the grass had a curiously bewildering effect on the eye. When the storm is over, the grass is found in drifts against the posts and wires of the fences; these collections remind one strongly of snow-drifts.

On December i6 (or so), I888, a terrible accident occurred on the railway between Candalaria and Guardia de la Esquina. A cutting had become filled with this Paja voladora; and the engine set fire to it as it passed. However, thanks mainly to a suitable wind, the train got safely through. But in the afternoon of the same day the train re-passed in the opposite direction, and the cutting had in the meantime become filled again with 